POS PROCEEDINGS

\title{
Upper limit for Poisson variable incorporating systematic uncertainties by Bayesian approach
}

Yongsheng Zhu*

Institute of High Energy Physics

Beijing 100049, China

E-mail: zhuys@ihep.ac.cn

\begin{abstract}
To calculate the upper limit for the Poisson observable at given confidence level with inclusion of systematic uncertainties in background expectation and signal efficiency, formulations have been established along the line of Bayesian approach. A FORTRAN program, BPULE, has been developed to implement the upper limit calculation.
\end{abstract}

XI International Workshop on Advanced Computing and Analysis Techniques in Physics Research April 23-27 2007

Amsterdam, the Netherlands

\footnotetext{
${ }^{*}$ Speaker.
} 


\section{Introduction}

A group of particle physics experiments involves the search for new signal or measuring small signal at the circumstance with significant background. A limit on, or a measurement of, a physical quantity at a given confidence level is usually set by comparing a number of detected events with the expected number of background events in the "signal" region where the signal events (if exist) shall reside. How well this comparison can be made for the observed events and the expected background depends strongly on the systematic uncertainties existing in the measurement. Therefore, systematic uncertainties must be taken into consideration in the limit or confidence belt calculation.

In the frame of frequentist statistics, confidence limits are set using a Neyman construction [1]. This method suffers from so-called undercoverage and "flip-flopping" policy when the observable is close to the physics boundary, namely, the actual coverage is less than the requested coverage (confidence level) and to report a central confidence interval or an upper limit is artificially decided by the experimenter's choice. In particular, in the case when no events have been observed, this method gives no answer for the confidence interval.

Feldman and Cousins [2] proposed a new method to construct confidence interval based on likelihood ratios, which automatically provides a central confidence interval or an upper confidence limit, which is decided by the observed data itself. Therefore, it is often denoted as the "unified approach".

However, this approach also has its drawbacks. If the observable is a Poisson variable, there is a background dependence of the upper limit in the case of fewer events observed than expected background. This can lead to situation where measurements with higher background give a smaller upper limit, which is clearly undesirable. To overcome this shortcoming, Roe and Woodroofe [3] proposed a solution to this problem by using such a fact that, given an observation $n$, the background $b$ can not be large than $n$ in any case. Therefore, the usual Poisson pdf (probability density function) should be replaced by a conditional pdf, and then this conditional pdf is used to construct the confidence interval. This approach solves the background dependence of the upper limit, however, does not satisfy all the requirements of proper coverage [4] and has problems when applied to the case of a Gaussian distribution with boundaries [5]. An extension based on a Bayesian approach with tests of coverage can be found in [6].

Along this line, a modification of the Neyman method incorporating systematic uncertainty of the signal detection efficiency has been proposed by Highland and Cousins [7], in which a "semiBayesian" approach is adopted, where an average over the probability of the detection efficiency is performed. This method is of limited accuracy in the limit of high relative systematic uncertainties. On the other hand, an entirely frequentist approach has been proposed for the uncertainty in the background rate prediction [8]. This approach is based on a two-dimensional confidence belt construction and likelihood ratio hypothesis testing and treats the uncertainty in the background as a statistical uncertainty rather than as a systematic one. Recently, Conrad etal extend the method of confidence belt construction proposed in [2] to include systematic uncertainties in both the signal and background efficiencies as well as systematic uncertainty of background expectation prediction [9]. It takes into account the systematic uncertainties by assuming a pdf which parameterizes our knowledge on the uncertainties and integrating over this pdf. This method, combining classical and Bayesian elements, is referred to as semi-Bayesian approach. A FORTRAN program, POLE, 
has been coded to calculate the confidence intervals for a maximum of observed events of 100 and a maximum signal expectation of 50 [10].

In the frame of Bayes statistics, Narsky depicted the estimation of upper limits for Poisson statistic with the known background expectation [11], [12]. Treatment of background uncertainty is discussed with the flat prior for simplified cases of background expectation distributions in Refs [13], [7].

In this paper, we use Bayesian approach to formulate the upper limit at given confidence level for the Poisson observable incorporating systematic uncertainties in the signal efficiency and background expectation. A FORTRAN program has been developed to calculate the corresponding upper limit.

\section{Bayesian approach to estimate upper limit}

In Bayesian approach one has to assume a prior pdf of an unknown parameter and then perform an experiment to update the prior distribution. The prior pdf reflects the experimenter's subjective degree of belief about unknown parameter before the measurement was carried out. The updated prior, called posterior pdf, is used to draw inference on unknown parameter. This updating is done with the use of Bayes theorem [14]. Assuming that $n$ represents the number of observed events, $s$ is the number of signal events which is unknown and to be inferred, $p(n \mid s)$ is the conditional pdf of observing $n$ events with given signal $s, \pi(s)$ is the prior pdf, the Bayes theorem gives the posterior pdf:

$$
h(s \mid n)=\frac{p(n \mid s) \pi(s)}{\int_{0}^{\infty} p(n \mid s) \pi(s) d s} .
$$

Here the lower limit of the integral is zero, which is the possible minimum number of signal events. Using this posterior pdf, one can calculate a Bayesian confidence interval for the signal expectation at given confidence level $C L=1-\alpha$ :

$$
1-\alpha=\int_{s_{L}}^{s_{U}} h(s \mid n) d s .
$$

The upper limit of the number of signal events at given confidence level $C L=1-\alpha, S_{U P}$, is naturally given by:

$$
1-\alpha=\int_{0}^{S_{U P}} h(s \mid n) d s .
$$

The nice feature of the Bayesian approach is that the zero value of an upper limit $S_{U P}$ always corresponds to the zero value of confidence level $C L=1-\alpha$, which is not necessarily true for the classical approach. The most important issue is to determine a prior pdf of the parameter. This is an issue which brings most of controversies into Bayesian methods. An important question is that if one should use an informative prior, i.e., a prior which incorporates results of previous experiments, or a non-informative prior, i.e., a prior which claims total ignorance. The major objection against informative prior is based on such argument: if we assume a prior which incorporates results of previous experiments, then our measurement will not be independent, hence, we will not be able to combine our results with previous results by taking a weighted average. Thus, we only discuss the Bayesian inference that assumes a non-informative prior for the non-negative parameter of a Poisson distribution. 
For the case that in the "signal region" where the signal events resides, the number of signal events is a Poisson variable with unknown expectation $s$, and the number of background events is a Poisson variable with expectation $b$, the conditional pdf of observing $n$ total events can be written as

$$
p(n \mid s)=e^{-(s+b)} \frac{(s+b)^{n}}{n !} .
$$

To deduce the posterior pdf, one has to assume a prior pdf. Bayes stated that, the non-informative prior for any parameter must be flat [14]. This statement does not based on any strict mathematical argument, but merely his intuition. The obvious weakness of Bayes prior pdf is that if one can assume a flat distribution of an unknown parameter, then one can also assume a flat distribution for any function of this parameter; however, these two prior functions are apparently not identical. Jeffreys [15], [16], Jaynes [17], and Box etal [18] derived the non-informative prior from first principle to resolve this problem, which are proportional to $1 / \theta$ and $1 / \sqrt{\theta}$, respectively, where $\theta$ is the unknown parameter. Comments on these three non-informative priors can be found in Refs. [11], [12]. For the pdf shown in Eq. 2.3, the corresponding prior pdfs are proportional to $1 /(s+b)$ and $1 / \sqrt{s+b}$. In general, we can use a prior pdf of

$$
\pi(s) \propto \frac{1}{(s+b)^{m}}, \quad s \geq 0, \quad 0 \leq m \leq 1,
$$

where $m=0$ corresponds to Bayes prior, $m=0.5$ to $1 / \sqrt{s+b}$ prior, and $m=1$ to $1 /(s+b)$ prior. One can choose $m$ value as he/she thinks appropriate, however, it should always be kept in mind that different $m$ value will give different answer for the confidence interval and upper limit. Expected coverage and length of confidence intervals constructed with these three priors and with the Neyman construction [1] and unified approach [2] can be found in Ref. [12]. It has been shown that the $1 / \sqrt{s+b}$ prior is the most versatile choice among the Bayesian methods, it provides a reasonable mean coverage for the confidence interval and upper limit for Poisson observable.

Substituting $p(n \mid s)$ of Eq. 2.3 and $\pi(s)$ of Eq. 2.4 into Eq. 2.1, the posterior pdf is then given by

$$
h(s \mid n)=\frac{(s+b)^{n-m} e^{-(s+b)}}{\Gamma(n-m+1, b)},
$$

where

$$
\Gamma(x, b)=\int_{b}^{\infty} s^{x-1} e^{-s} d s, \quad x>0, b>0
$$

is an incomplete gamma function.

In the case that the systematic uncertainties of the signal efficiency and background expectation can be neglected, the signal expectation $s$ is an unknown constant and the background expectation $b$ is a known value. Substituting this posterior pdf into Eq. 2.2, we obtain

$$
\alpha=\frac{\Gamma\left(n-m+1, S_{U P}+b\right)}{\Gamma(n-m+1, b)} .
$$

If the flat prior $(m=0)$ is used, Eq. 2.7 turns into

$$
\alpha=e^{-S_{U P}} \cdot \frac{\sum_{k=0}^{n} \frac{\left(S_{U P}+b\right)^{k}}{k !}}{\sum_{k=0}^{n} \frac{b^{k}}{k !}} .
$$


The upper limit $S_{U P}$ at a given confidence level $1-\alpha$ can be acquired by solving Eq. 2.7 or Eq. 2.8 numerically from measured values of $n$ and $b$.

Now we turn to the question of inclusion of systematic uncertainties.

First we consider only the uncertainty of background expectation is present, and the distribution of the background expectation is represented by a pdf $f_{b^{\prime}}\left(b, \sigma_{b}\right)$ with the mean $b$ and standard deviation $\sigma_{b}$. The conditional pdf expressed by Eq. 2.3 now is modified to

$$
q(n \mid s)_{b}=\int_{0}^{\infty} p(n \mid s)_{b^{\prime}} \cdot f_{b^{\prime}}\left(b, \sigma_{b}\right) d b^{\prime},
$$

where $p(n \mid s)_{b^{\prime}}$ has the same expression in Eq. 2.3 with $b$ replaced by $b^{\prime}$.

Next we take into account the uncertainties of the signal efficiency and background expectation simultaneously, and consider they are independent each other. The distribution of the signal relative efficiency $\varepsilon$ (with respect to the mean value of the signal detection efficiency) is described by a pdf $f_{\varepsilon}\left(1, \sigma_{\varepsilon}\right)$ with the mean 1 and standard deviation $\sigma_{\varepsilon}$. The conditional pdf described by Eq. 2.3 is then further modified to

$$
q(n \mid s)_{b}=\int_{0}^{\infty} \int_{0}^{\infty} p(n \mid s \varepsilon)_{b^{\prime}} f_{b^{\prime}}\left(b, \sigma_{b}\right) f_{\varepsilon}\left(1, \sigma_{\varepsilon}\right) d b^{\prime} d \varepsilon,
$$

where $p(n \mid s \varepsilon)_{b^{\prime}}$ represents that $b$ is replaced by $b^{\prime}$, and $s$ by $s \varepsilon$ in Eq. 2.3 . One notices that the lower limits of integrals in Eqs. 2.9, 2.10 are all zeros, which are the possible minimum value of any efficiencies and number of background events. Using $q(n \mid s)_{b}$ in Eqs. 2.9, 2.10 to construct posterior pdf

$$
h(s \mid n)=\frac{q(n \mid s)_{b} \pi(s)}{\int_{0}^{\infty} q(n \mid s)_{b} \pi(s) d s},
$$

one can calculate the upper limit $S_{U P}$ on $s$ at any given confidence level with inclusion of systematic uncertainties in terms of Eq. 2.2

\section{BPULE: An algorithm for calculating confidence upper limit}

We have developed an algorithm to calculate the upper limit for the Poisson observable at given confidence level with or without inclusion of systematic uncertainties in background expectation and signal efficiency. It has been implemented as a FORTRAN program, BPULE (Bayesian Poissonian Upper Limit Estimator) [19], where an iterative procedure is carried out by minimizing the difference between the given confidence level and the calculated value in terms of Eq. 2.2 until a convergence is reached. It contains two executable files: BPULE.exe for calculating a single upper limit while BPULE_batch.exe for a batch of upper limits. When the code is implemented, a flag is required to input, indicating what kind of upper limit (UL) is going to be calculated. Four options are available: UL without inclusion of any systematic uncertainty, UL with inclusion of merely systematic uncertainty of background expectation and merely systematic uncertainty of signal efficiency, and UL with inclusion of systematic uncertainties of both signal efficiency and background expectation, which are considered to be independent each other. There are also flags to select the type of distribution for detection efficiency and/or background expectation. Three types 
of functions with the mean 1 and standard deviation $\sigma$ are supported: Gaussian, Log-Gaussian and flat distributions. For the Log-Gaussian distribution, the pdf is

$$
\frac{1}{\sqrt{2 \pi} \sigma_{x} x} e^{\frac{-(l n x-\mu)^{2}}{2 \sigma_{x}^{2}}}, \quad x \in(0, \infty)
$$

where $\mu=-\ln \left(1+\sigma^{2}\right) / 2, \sigma_{x}=\sqrt{\ln \left(1+\sigma^{2}\right)}$.

\section{BPULE's results and discussions}

The upper limits for some typical cases have been calculated with BPULE. Inclusion of systematic uncertainties generally leads to a larger upper limit. We restrict to presenting the $S_{U P}$ varying with systematic uncertainty of signal and background efficiency separately to give a clear idea of the effect of a single variable at a time. Real applications usually combine those uncertainties.

\subsection{Comparison of results of BPULE and POLE}

Examples for some resultant upper limits acquired by BPULE and POLE [9] are given in Table 1 and 2. Different combinations of the number of measured events $n$ and the expected background $b$ are given for different uncertainties in signal efficiency and background expectation. The results of BPULE are obtained under flat and $1 / \sqrt{s+b}$ prior with Gaussian pdf for uncertainty distributions. There are differences between the results given by BPULE and POLE for same $n$ and $b$, this reflects that the confidence intervals and upper limits at a same nominal confidence level determined by the Bayesian approach and classical/semi-Bayesian approach virtually differ from each other [12]. Even so, some common trends can be found: the inclusion of systematic uncertainties leads to an increase of the confidence upper limit; when the uncertainty of background expectation increases, the upper limit grows only gently, while it grows much faster with increased uncertainty of signal efficiency.

\subsection{Comparison of results of flat and $1 / \sqrt{s+b}$ priors}

The upper limits determined by $1 / \sqrt{s+b}$ prior are smaller than those by flat prior as can be seen from Table 1, 2 and 3, where $S_{U P}(0.9)$ (upper limit at 90\% CL) for $n=b=0,1, \cdots, 10$ is presented without inclusion of systematic uncertainty. The reason is that, the denominator in Eq. 2.1 just plays a role of a normalization factor, while the numerator determines the shape of the posterior pdf $h(s \mid n)$. In the flat prior case, $p(n \mid s)$ at any $s$ has same weight in $h(s \mid n)$, whereas in the $1 / \sqrt{s+b}$ or $1 /(s+b)$ prior case, $p(n \mid s)$ at larger $s$ has smaller weight, which induces the $h(s \mid n)$ value moving towards to small $s$. As a result, for a same confidence level, the bigger the $m$ value, the smaller the $S_{U P}$.

In case of fewer events observed than background expectation $(n<b)$, the $S_{U P}(0.9)$ versus $b$ at $n=0$ without inclusion of systematic uncertainties using $1 / \sqrt{s+b}$ prior is listed in Table 4; while the $S_{U P}(0.9)$ would be all equal to 2.30 for any $b>0$ at $n=0$ if we use flat prior.

Although the Bayesian flat prior has been widely used to determine the upper limit, however, as stated in section 2 , from the Bayesian statistics point of view, the $1 / \sqrt{s+b}$ prior may be more appropriate. 
Table 1: $S_{U P}(0.9)$ (Upper limit at 90\% CL) incorporating systematic uncertainty in signal efficiency which pdf is Gaussian with mean 1 and standard deviation $\sigma_{\varepsilon}$. The results of POLE are quoted from Ref. [9].

\begin{tabular}{|l|c|c|c|c|c|c|}
\hline$n$ & $b$ & $\sigma_{\varepsilon}$ & \multicolumn{2}{|c|}{ POLE } & \multicolumn{2}{c|}{ BPULE } \\
\cline { 4 - 7 } & & & $\begin{array}{c}\text { Likelihood } \\
\text { ratio }\end{array}$ & $\begin{array}{c}\text { Likelihood ratio } \\
\text { with conditioning }\end{array}$ & $\begin{array}{c}\text { flat } \\
\text { prior }\end{array}$ & $\begin{array}{c}1 / \sqrt{s+b} \\
\text { prior }\end{array}$ \\
\hline 2 & 2 & 0. & 3.90 & 4.00 & 3.88 & 3.41 \\
& & 0.1 & & & 3.97 & 3.48 \\
& & 0.2 & 3.95 & 4.34 & 4.32 & 3.71 \\
& & 0.3 & 4.10 & 4.75 & 5.57 & 4.37 \\
& & 0.4 & 4.65 & 5.35 & 9.87 & 5.94 \\
\hline 3 & 2 & 0. & 5.40 & 5.30 & 4.93 & 4.38 \\
& & 0.1 & & & 5.05 & 4.47 \\
& & 0.2 & 5.70 & 5.65 & 5.52 & 4.79 \\
& & 0.3 & 5.95 & 6.20 & 7.19 & 5.65 \\
& & 0.4 & 6.80 & 7.10 & 13.3 & 7.69 \\
\hline 4 & 2 & 0. & 6.60 & 6.60 & 6.09 & 5.50 \\
& & 0.1 & & & 6.25 & 5.61 \\
& & 0.2 & 7.10 & 7.30 & 6.87 & 6.04 \\
& & 0.3 & 7.75 & 7.85 & 9.05 & 7.16 \\
& & 0.4 & 8.95 & 9.15 & 17.2 & 9.78 \\
\hline
\end{tabular}

\subsection{Comparison of results for three type pdfs for uncertainties}

Fig. 1 shows, for $n=10, b=8$, the $S_{U P}(0.9)$ as function of $\sigma_{b}^{\prime}\left(=\sigma_{b} / b\right)$ and $\sigma_{\varepsilon}$ for three type pdfs (Gaussian, Log-Gaussian, and flat) of uncertainties using flat and $1 / \sqrt{s+b}$ prior, respectively. The relations of $S_{U P}(0.9)$ versus $\sigma_{b}^{\prime}$ for flat and Gaussian pdfs are similar, and the difference with that of Log-Gaussian pdf is also small. The $S_{U P}(0.9)$ grows faster for Gaussian pdf than those of flat and Log-Gaussian pdfs with increased $\sigma_{\varepsilon}$, especially in larger $\sigma_{\varepsilon}$; in particular when the flat prior is used the $S_{U P}(0.9)$ rises drastically. This picture is typical for flat prior and Gaussian pdf in larger $\sigma_{\varepsilon}$, which warns us that in this case, cautious consideration must be taken that if the Gaussian pdf is a proper distribution for signal efficiency uncertainty.

\subsection{Relation of $S_{U P}$ vs. $b$ without systematic uncertainty}

Fig.2 shows the $S_{U P}(0.9)$ as function of $b$ at different values of $n-b$ without inclusion of systematic uncertainty, which are calculated with flat and $1 / \sqrt{s+b}$ priors, respectively. It can be seen that when enlarge the "signal region"( $b$ will be increased, and $n-b$ will be nearly constant if the signal region is wide enough to contain the signal events), $S_{U P}(0.9)$ will increase slowly except both $n$ and $b$ close to zeros. For example, for $b=10,20$ and $n-b=8$, the corresponding $S_{U P}(0.9)$ with flat prior are 14.8 and 16.2, respectively. This means that, enlarging the width of the signal region will not significantly change the $S_{U P}(0.9)$. 
Table 2: $S_{U P}(0.9)$ incorporating systematic uncertainty in background expectation which pdf is Gaussian with mean $b$ and standard deviation $\sigma_{b}=b \sigma_{b}^{\prime}$. The results of POLE are quoted from Ref. [9].

\begin{tabular}{|l|c|c|c|c|c|c|}
\hline$n$ & $b$ & $\sigma_{b}^{\prime}$ & \multicolumn{2}{|c|}{ POLE } & \multicolumn{2}{c|}{ BPULE } \\
\cline { 4 - 7 } & & & $\begin{array}{c}\text { Likelihood } \\
\text { ratio }\end{array}$ & $\begin{array}{c}\text { Likelihood ratio } \\
\text { with conditioning }\end{array}$ & $\begin{array}{c}\text { flat } \\
\text { prior }\end{array}$ & $\begin{array}{c}1 / \sqrt{s+b} \\
\text { prior }\end{array}$ \\
\hline 2 & 2 & 0. & 3.90 & 4.00 & 3.88 & 3.41 \\
& & 0.1 & & & 3.89 & 3.43 \\
& & 0.2 & 3.95 & 4.10 & 3.93 & 3.46 \\
& & 0.3 & 3.95 & 4.25 & 4.00 & 3.53 \\
& & 0.4 & 3.95 & 4.35 & 4.07 & 3.60 \\
\hline 3 & 2 & 0. & 5.40 & 5.30 & 4.93 & 4.38 \\
& & 0.1 & & & 4.94 & 4.40 \\
& & 0.2 & 5.45 & 5.35 & 4.98 & 4.44 \\
& & 0.3 & 5.45 & 5.45 & 5.05 & 4.51 \\
& & 0.4 & 5.50 & 5.55 & 5.13 & 4.58 \\
\hline 4 & 2 & 0. & 6.60 & 6.60 & 6.09 & 5.50 \\
& & 0.1 & & & 6.10 & 5.51 \\
& & 0.2 & 6.95 & 6.65 & 6.14 & 5.55 \\
& & 0.3 & 6.95 & 6.80 & 6.20 & 5.60 \\
& & 0.4 & 6.95 & 6.80 & 6.27 & 5.67 \\
\hline
\end{tabular}

Table 3: $S_{U P}(0.9)$ for $n=b=0,1, \cdots, 10$ without inclusion of systematic uncertainty.

\begin{tabular}{|c|ccccccccccc|}
\hline$n=b=$ & 0 & 1 & 2 & 3 & 4 & 5 & 6 & 7 & 8 & 9 & 10 \\
\hline $\begin{array}{c}\text { Flat } \\
\text { prior }\end{array}$ & 2.30 & 3.27 & 3.88 & 4.36 & 4.78 & 5.15 & 5.49 & 5.80 & 6.09 & 6.37 & 6.63 \\
\hline $\begin{array}{c}1 / \sqrt{s+b} \\
\text { prior }\end{array}$ & 1.35 & 2.76 & 3.41 & 3.92 & 4.36 & 4.74 & 5.09 & 5.41 & 5.70 & 5.98 & 6.25 \\
\hline
\end{tabular}

Table 4: $S_{U P}(0.9)$ determined by $1 / \sqrt{s+b}$ prior for $n=0$ and $b=0,1, \cdots, 10$ without inclusion of systematic uncertainty.

\begin{tabular}{|c|ccccccccccc|}
\hline$b$ & 0 & 1 & 2 & 3 & 4 & 5 & 6 & 7 & 8 & 9 & 10 \\
\hline$S_{U P}(0.9)$ & 1.35 & 1.92 & 2.03 & 2.08 & 2.12 & 2.15 & 2.16 & 2.18 & 2.19 & 2.21 & 2.21 \\
\hline
\end{tabular}

\subsection{Relation of $S_{U P}$ vs. $b$ incorporating systematic uncertainties}

Fig.3 shows the $S_{U P}(0.9)$ calculated with flat prior as function of $b$ at different values of $n-b$ incorporating systematic uncertainties of background expectation and signal efficiency, $\sigma_{b}^{\prime}, \sigma_{\varepsilon}$. The pdfs of systematic uncertainties of background expectation and signal efficiency are assumed to be Gaussians. When the systematic uncertainty of signal efficiency is taken into account, the $S_{U P}(0.9)$ is not significantly sensitive to the width of "signal region", similar to the case of without inclusion of systematic uncertainty. However, this situation does not hold when the systematic uncertainty 
of background expectation is taken into consideration. The bigger the $\sigma_{b}^{\prime}$, the faster the $S_{U P}(0.9)$ grows for increased $b$.

\subsection{Relation of $S_{U P}$ vs. $\sigma_{b}^{\prime}, \sigma_{\varepsilon}$ incorporating systematic uncertainties}

Fig.4 and 5 show the $S_{U P}(0.9)$ as functions of $\sigma_{b}^{\prime}$ and $\sigma_{\varepsilon}$ at different $b$ values with inclusion of systematic uncertainties calculated with flat prior. The pdfs of systematic uncertainties of background expectation and signal efficiency are assumed to be Gaussians. For small $b$ value, the $S_{U P}(0.9)$ is insensitive to the variation of $\sigma_{b}^{\prime}$, while for larger $b$ the increase of the $S_{U P}(0.9)$ is gradually drastic with increased $\sigma_{b}^{\prime}$. The relations of $S_{U P}(0.9)$ vs. $\sigma_{\varepsilon}$ at different $b$ values are similar: the $S_{U P}(0.9)$ rises gently at small $\sigma_{\varepsilon}$, and rapidly for $\sigma_{\varepsilon} \geq 0.3$.

\subsection{Relation of $S_{U P}$ vs. $\sigma_{b}^{\prime}, \sigma_{\varepsilon}$ incorporating systematic uncertainties in case of $n<b$}

Fig.6 shows the $S_{U P}(0.9)$ as functions of $\sigma_{b}^{\prime}$ and $\sigma_{\varepsilon}$ in case of fewer events observed than background expectation incorporating systematic uncertainties, calculated with both flat and $1 / \sqrt{s+b}$ priors. The pdfs of systematic uncertainties of background expectation and signal efficiency are assumed to be Gaussians. The variation of $S_{U P}(0.9)$ with increased $\sigma_{b}^{\prime}$ is smooth in both priors,

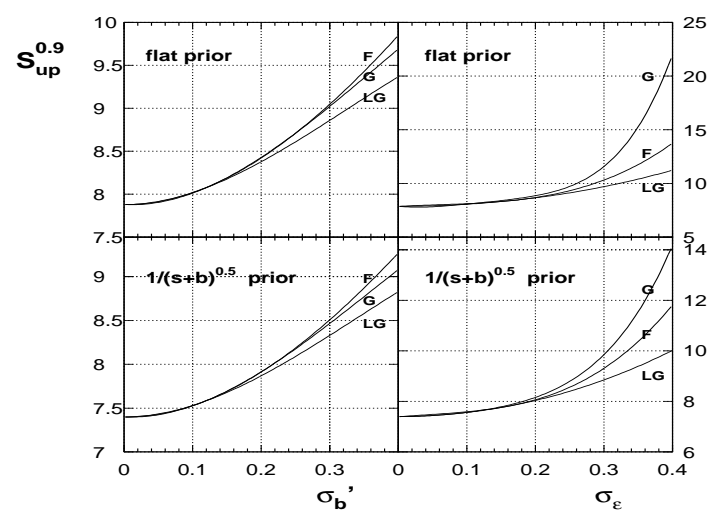

Figure 1: $S_{U P}(0.9)$ as functions of $\sigma_{b}^{\prime}$ and $\sigma_{\varepsilon}$ for $n=10, b=8$ and 3 type pdfs of uncertainties. (G: Gaussian, LG: Log-Gaussian, F: Flat.)

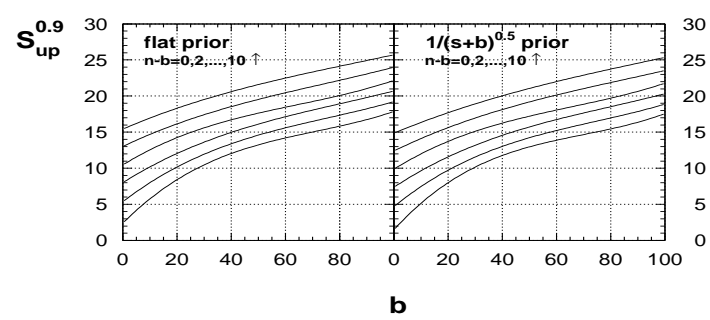

Figure 2: $S_{U P}(0.9)$ as function of $b$ without inclusion of systematic uncertainty. The curves are given for values of $n-b=0,2,4,6,8,10$, starting from the bottom, as indicated by the arrow in the figure. The same convention has been used in figures 3-5.

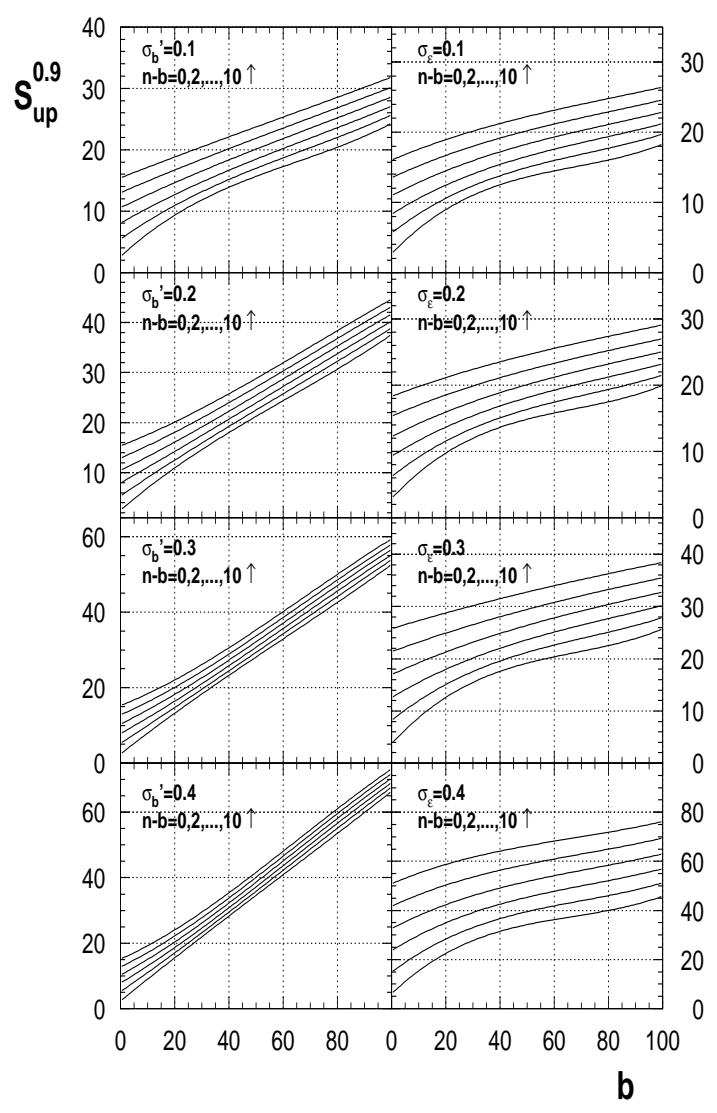

Figure 3: $S_{U P}(0.9)$ as function of $b$ incorporating Gaussian systematic uncertainties for background expectation with flat prior. 


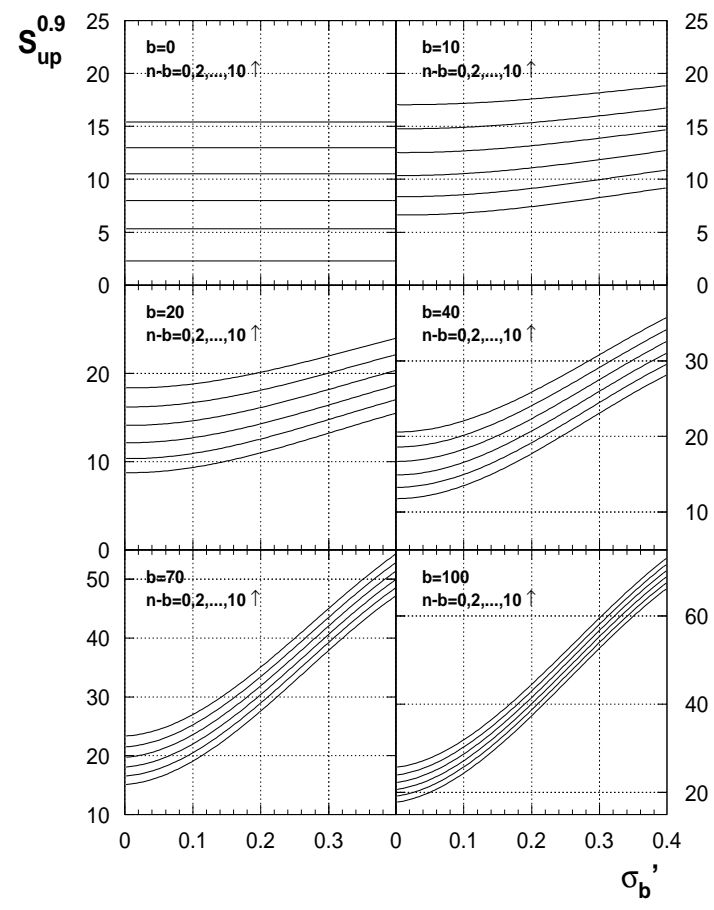

Figure 4: $\quad S_{U P}(0.9)$ as function of $\sigma_{b}^{\prime}$ incorporating Gaussian systematic uncertainty for background expectation with flat prior.

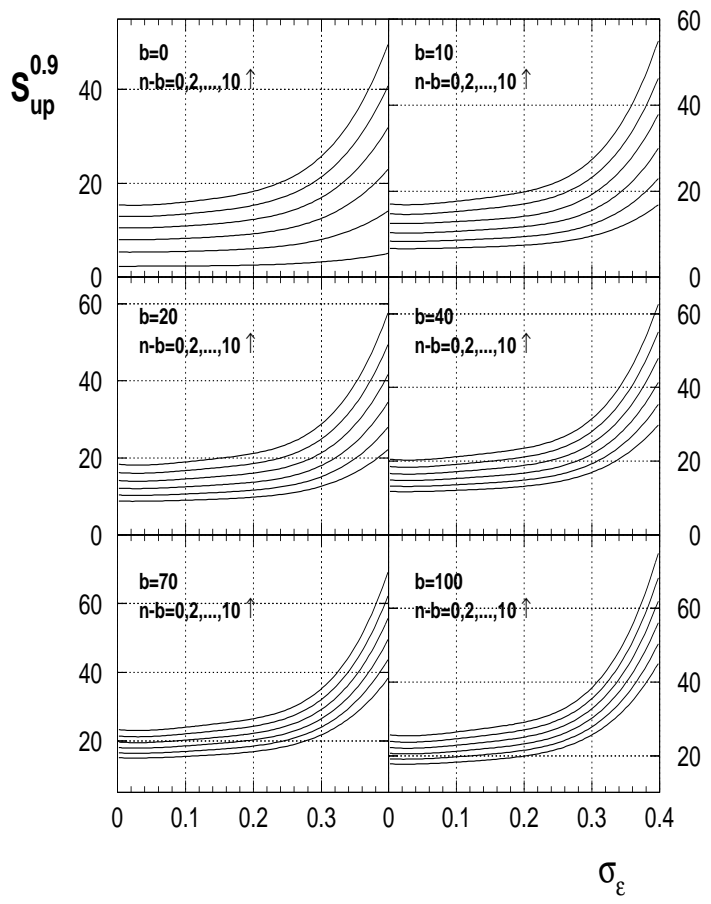

Figure 5: $\quad S_{U P}(0.9)$ as function of $\sigma_{\varepsilon}$ incorporating Gaussian systematic uncertainty for signal efficiency with flat prior.

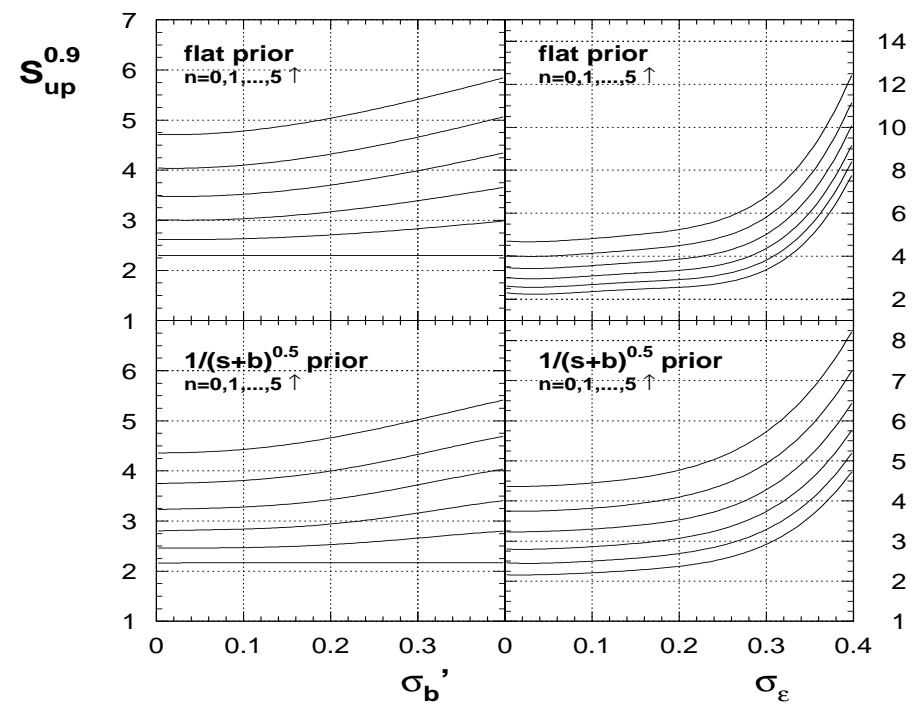

Figure 6: $S_{U P}(0.9)$ as functions of $\sigma_{b}^{\prime}$ and $\sigma_{\varepsilon} \cdot(b=6)$. The curves are given for values of $n=0,1,2,3,4,5$, starting from the bottom, as indicated by the arrow in the figure. 
while the $S_{U P}(0.9)$ rises gently at small $\sigma_{\varepsilon}$, and rapidly for $\sigma_{\varepsilon} \geq 0.3$, in particular when the flat prior is used.

\section{Summary}

We have formulated the upper limit calculation at any given confidence level in the frame of Bayesian approach for the Poisson observable incorporating systematic uncertainties in both the signal efficiency and background expectation prediction. A FORTRAN program, BPULE, has been developed to implement the upper limit calculation. Generally, the inclusion of systematic uncertainties leads to an increase of the confidence upper limit. The upper limits for some typical cases have been calculated with BPULE and related discussions have been presented.

\section{Acknowledgments}

This work is supported in part by the National Natural Science Foundation of China under contracts Nos. 19991483 and 10491303. The author gratefully acknowledges the helps provided by Dr. LI Gang in programming and by Dr. MO Xiaohu and WANG Youkai in figures drawing.

\section{References}

[1] J. Neyman, Philos. Trans. R. Soc. London, Series A,236, 333(1937).

[2] G.J. Feldman and R.D. Cousins, Phys. Rev. D57, 3873 (1998).

[3] B.P. Roe and M.B. Woodroofe, Phys. Rev. D60, 053009 (1999).

[4] G. Zech, hep-ex/0106023.

[5] R.D. Cousins, Phys. Rev. D62, 098301 (2000)

[6] B.P. Roe and M.B. Woodroofe, Phys. Rev. D63, 013009 (2001).

[7] R.D. Cousins and V.L. Highland, Nucl. Instrum. Methods A320,331 (1992)

[8] W.A. Rolke and A.M. Lopez, Nucl. Instrum. Methods A458,745 (2001).

[9] J. Conrad etal., Phys. Rev. D67, 012002 (2003).

[10] http://www3.tsl.uu.se/ conrad/pole.html.

[11] I. Narsky, Nucl. Instrum. Methods A450, 444 (2000).

[12] I. Narsky, arXiv:hep-ex/0005019 (2000).

[13] O. Helene, Nucl. Instrum. Methods A300, 132 (1991).

[14] T. Bayes, Phil. Trans. Roy. Soc., 53, 370 (1763)

[15] H. Jeffreys, Theory of probability, 3rd Edition, Clarendon. Oxford, 1961.

[16] H. Jeffreys, Proc. of the Royal Soc. of London, A 196, 453 (1946).

[17] H. Jaynes, IEEE Trans. on System Sci. and Cyber. SSC-4, 3, 227 (1968).

[18] G.E.P. Box and G.C. Tiao, Bayesian Inference in Statistical Analysis, Wiley Classics, 1992.

[19] http://www.ihep.ac.cn/lunwen/zhuys/BPULE/BPULE.html 